\title{
Optical Power Transmission Through Adhesive and Bonding Layers
}

\author{
Arsen V. Subashiev and Serge Luryi, Fellow, IEEE
}

\begin{abstract}
In this paper, we analyze the optical power transmission in structures that include a low-index intermediate layer and sources with a wide angular distribution. Special attention is paid to the angular average of the transmission coefficient, which can be cast in a universal form for two practically relevant classes of source layers. Due to the so-called frustrated total internal reflection, the structure transparency is highly sensitive to the intermediate layer thickness and index contrast. We show that the transmission coefficient for isotropic radiation may remain low even for optically thin low-index intermediate layers, so that the usual comparison between the optical thickness and the wavelength is no longer a reliable criterion. Calculations are presented for exemplary structures, such as a semiconductor scintillator bonded to a photodiode. The angular dependence of the transmission coefficient is shown to satisfy a simple and universal sum rule.
\end{abstract}

Index Terms-Emission efficiency, light emitting diodes, luminescence, semiconductor scintillators.

\section{INTRODUCTION}

A number of semiconductor optoelectronic devices include light-emitting sources that produce monochromatic radiation isotropically distributed in a wide range of emission angles. In the design of such structures, one is often concerned with the average optical power transmission across the boundaries of the source layer. For example, the external emission efficiency of semiconductor LEDs is limited to $2 \%-4 \%$ unless special precautions are taken. The low external efficiency arises from the high refractive index of the source material, resulting in a narrow escape angle against total internal reflection (TIR) [1]. To prevent the TIR, various techniques have been used, including random surface texturing, pyramidal-shaped structures, as well as devices exploiting "wave optics" effects and optimizing interference in the resonant cavity (see [2] for the review). The most effective remedy for the TIR effects in LEDs is to use an optically tight lens, resulting in external emission efficiencies approaching unity [3], [4]. Another example of interest to us is semiconductor scintillator, integrated with a pho-

Manuscript received April 28, 2009; revised July 16, 2009 and August 19, 2009. First published September 01, 2009; current version published October 07, 2009. This work was supported in part by the Domestic Nuclear Detection Office of the Department of Homeland Security, by the Defense Threat Reduction Agency through its basic research program, and by the New York State Office of Science, Technology and Academic Research through the Center for Advanced Sensor Technology at Stony Brook.

The authors are with the Department of Electrical and Computer Engineering, State University of New York at Stony Brook, Stony Brook, NY 11794-2350

USA (e-mail: subashiev@ece.sunysb.edu; serge.luryi@stonybrook.edu).

Color versions of one or more of the figures in this paper are available online at http://ieeexplore.ieee.org.

Digital Object Identifier 10.1109/JLT.2009.2031309 todiode to register the scintillating radiation [5]. Ionizing interaction with a high-energy particle gives rise to an isotropic emission source within the scintillator slab, and the quantification of the generated luminescent signal strongly depends on its transmission across the slab boundary.

In both cases, the optical components can be attached by using optical adhesives or optical glues in the form of a thin interlayer. The refractive index of the interlayer is typically considerably lower than that of both semiconductors. It is usually expected that films with optical thickness smaller than the wavelength should not disturb the radiation transmission or waveguiding properties [6]. This is indeed true for interlayers of higher refractive index. However, for low-index interayers and an isotropic source of radiation, the angles of incidence are widely spread, and a considerable part of incident radiation is in the TIR region for the semiconductor-interlayer interface. Transmission of this part of radiation across a thin interlayer is provided by evanescent optical waves and decays exponentially with the interlayer thickness. The residual transparency is known as the frustrated TIR or FTIR. ${ }^{1}$

Due to the TIR phenomenon, the average transmission of isotropic radiation may remain low even for optically thin lowindex interlayers, and the usual comparison between the optical thickness and the wavelength is no longer a reliable guide. To be sure, the comparison remains a valid criterion for normal incidence. Moreover, for a monochromatic source and any fixed angle of incidence, the transparency can be generally enhanced by choosing the thickness of the low-index layer, so as to suppress the reflected wave using wave interference and make the interlayer an "absentee" layer. But this does not work for widely distributed angles of incidence spread over the FTIR region. The average transparency remains dependent on the layer index profile, but the requirements on the intermediate layer thickness become much more restrictive.

This paper begins with an analysis of the average power transmission of isotropic radiation resulted from an emission source distributed in a high-index layer and transmitted through a thin lower index film. It turns out that the transmitted optical power can be described by an angle-averaged transmission coefficient, but the very definition of the averaging procedure depends on whether the source layer is transparent or absorbing. We show that in both limits, the average transmission coefficients can be unambiguously defined and proceed to calculate these coefficients for exemplary structures. Finally, we discuss the stringent requirements on the interlayer thickness to ensure high average transmission.

\footnotetext{
${ }^{1} \mathrm{~A}$ review of the history and applications of FTIR can be found in [7].
} 


\section{ANgUlar AVERAge OF POWER TrAnSMission}

In a general structure with low-index interlayer, the transmitted radiation may strongly depend on the details of geometry, especially when the linear dimensions of the emitting region are comparable to the wavelength or the absorption length. In this situation, one may need detailed modeling, taking into account the boundary effects that are nonuniform laterally. However, when the lateral size of the structure is much larger than either the absorption length or the linear dimensions of the source, the lateral nonuniformity of boundary conditions becomes negligible. Fortunately, this is the case most relevant for applications to semiconductor optoelectronic devices in the visible and near-IR range.

Consider first a nonmagnetic transparent isotropic layer of thickness $\Delta$ with a point-like isotropic source of power $P$ located at a distance $z$ from the layer surface. The normal component of the incident flux through an infinitesimal circular annulus of radius $\rho=z / \tan \phi_{1}$ and area $d s=2 \pi \rho d \rho$ is $d I_{z=0}=$ $P /\left(4 \pi r^{2}\right) \cos \phi_{1} d s=P /(4 \pi) d \Omega$. Here, $\phi_{1}$ is the incidence angle, $r=z / \cos \phi_{1}$, and $d \Omega=2 \pi \sin \phi_{1} d \phi_{1}$ is the solid angle of illumination of area $d s$. We shall consider the wave propagation in the source layer within the limits of geometrical optics, $\lambda \ll \Delta$, which allows us to neglect interference effects in emission. Then, for an interface with a transmission coefficient $T\left(\phi_{1}\right)$, the total transmitted power can be written as

$$
P_{t}=\frac{1}{2}\langle T\rangle P
$$

where the average transmission coefficient is defined by

$$
\langle T\rangle=\frac{1}{2} \int_{0}^{\pi / 2}\left[T_{s}\left(\phi_{1}\right)+T_{p}\left(\phi_{1}\right)\right] \sin \phi_{1} d \phi_{1}
$$

with $T_{s}\left(\phi_{1}\right)$ and $T_{p}\left(\phi_{1}\right)$ being the transmission coefficients for two polarizations of the incident radiation. ${ }^{2}$

Note that $P_{t}$ does not depend on the source location $z$. Therefore, one can use the same equations to describe the power transmitted through a small surface patch of unit area from a distributed unpolarized ${ }^{3}$ source of large lateral extent. If the volume density of emitted power in the distributed source is $p$, then replacing $P \rightarrow p \Delta$ on the right-hand side of (1) gives the average transmitted power per unit area.

Definition (2) corresponds to the simple averaging of the transmission coefficient over the solid angle of incident radiation. It can be used to estimate the external efficiency of LED and scintillators when the source layer is transparent, i.e., its thickness $\Delta$ is thinner than the absorption length, $\Delta \ll \alpha^{-1}$, where $\alpha$ is the source layer absorption coefficient. ${ }^{4}$

\footnotetext{
${ }^{2}$ We use the subscript $s$ for $s$-polarized waves (electric field perpendicular to the plane of incidence) and $p$ for $p$-polarized waves.

${ }^{3}$ For a source layer composed of quantum wells, the emission may strongly differ for $s$ - and $p$-polarized waves. This case becomes sensitive to the polarization distribution and requires special analysis.

${ }^{4}$ Equations (1) and (2) do not apply directly to the situation when the source layer is equipped with a back mirror, even within the approximation of geometrical optics. Reflection from the back surface would enhance the power transmission and alter its angular dependence by offering reflected photons another chance.
}

In the opposite case of radiation escaping from a source embedded in an absorbing layer, the incidence angle distribution is not universal. Indeed, for a point-like source at a distance $z$ from the surface, we have

$$
\begin{aligned}
d I_{z=0} & =\frac{P}{4 \pi r^{2}} e^{-\alpha r} \cos \phi_{1} d s \\
& =\frac{P}{2} e^{-\alpha z / \cos \phi_{1}} \sin \phi_{1} d \phi_{1}
\end{aligned}
$$

so that for a source at $z>1 / \alpha$, the angular distribution is narrowed. The narrowing increases with $z$. For nonmonochromatic sources in dispersive media, $\alpha=\alpha(\omega)$, the angular dependence becomes even more complicated.

A simplified angular distribution emerges in the limiting case of a homogeneously distributed source, whose linear dimensions (including the thickness) much exceed the absorption length. In this case, every small part of the surface is illuminated by an isotropic flux coming from distances within the absorption length. The common example of such a source is the quasi-equilibrium interband luminescence of an optically (or electrically) excited semiconductor layer when the excitation region is much larger than the absorption length.

Consider the total power emitted from an absorbing layer with the density of emitters $p$. Integrating (3) over $z$, for the differential flux per unit area, we find

$$
d I_{z=0}=\frac{p}{2 \alpha}\left[1-\exp \left(-\frac{\alpha \Delta}{\cos \phi_{1}}\right)\right] \cos \phi_{1} \sin \phi_{1} d \phi_{1}
$$

For a thick source layer, $\alpha \Delta \gg 1$, the exponential term in (4) becomes negligible. This leads to a universal angular distribution of the flux at the surface. The appropriate average transmission coefficient in this case is of the form

$$
\langle T\rangle_{\mathrm{eq}}=\int_{0}^{\pi / 2}\left[T_{s}\left(\phi_{1}\right)+T_{p}\left(\phi_{1}\right)\right] \cos \phi_{1} \sin \phi_{1} d \phi_{1}
$$

so that the transmitted power per unit area is

$$
P_{t}=\frac{p}{4 \alpha}\langle T\rangle_{\mathrm{eq}} .
$$

Definition (5) is commonly used in the discussion of equilibrium blackbody radiation (see, e.g., [8] and Appendix I).

Note that both averages (2) and (5) are normalized so that for a nonreflecting boundary, one has $\langle T\rangle_{\mathrm{eq}}=\langle T\rangle=1$. This requires an additional factor of 2 in (5).

For nonequilibrium sources, the averaging procedure (5) has been applied to the situation when photon recycling dominates the source properties [9]. In this case, due to multiple photon absorption/reemission processes, the radiation may be widely distributed over the source layer. In general, however, the situation that takes place in common photoluminescence experiments may conform either to (2) or (5), depending on the re- 
lation between the minority carrier diffusion length $L$ and the absorption length $\alpha^{-1}$ for outgoing light. ${ }^{5}$

It should be stressed that the two averaging procedures lead to tangible differences in power transmission only when the radiation goes through a large solid angle. When the transmission is restricted by the TIR to small angles (as is the case for emission from a semiconductor to low-index media, such as air or vacuum), the difference is minor. Indeed, one can compare (1) and (6) for similarly (homogeneously) distributed sources. For small-angle restricted transmission, one has $\langle T\rangle_{\mathrm{eq}} \approx 2\langle T\rangle$, so that (6) reads $P_{t}=p /(2 \alpha)\langle T\rangle$. Thus, the transmitted power is proportional to half of the power emitted in a layer with thickness $\Delta=1 / \alpha$, in agreement with (1). The difference in the average power transmission using either $\langle T\rangle_{\mathrm{eq}}$ or $\langle T\rangle$ becomes negligible. ${ }^{6}$ For emission into vacuum from a semiconductor with $n \approx 4$, the transmitted fraction of optical power can be estimated as $1 / 2 n^{2}$ for both averaging procedures. However, for transmission not restricted to small angles (e.g., for low-index contrast), the difference becomes substantial. Thus, for a nonreflective surface, $P_{t}=p /(4 \alpha)$, which is half that predicted by (1).

For the average transmission coefficient $\langle T\rangle_{\mathrm{eq}}$ (but not for $\langle T\rangle$ ), a universal reciprocity relation can be proven viz.

$$
\left.n_{1}^{2}\langle T\rangle_{\mathrm{eq}}\right|_{1 \rightarrow 3}=\left.n_{3}^{2}\langle T\rangle_{\mathrm{eq}}\right|_{3 \rightarrow 1} .
$$

This relation follows from a general thermodynamic equilibrium argument (see Appendix I), and it takes the form of a universal sum rule satisfied by the transmission coefficient for any index and arbitrary thickness of the interlayer, as well as for each polarization mode individually ${ }^{7}$

$$
n_{1}^{2} \int T_{13}(\phi) \cos (\phi) d \Omega=n_{3}^{2} \int T_{31}(\phi) \cos (\phi) d \Omega .
$$

In particular, for low-index interlayers, the integration in (8) includes the FTIR range of angles. To be sure, (8) does not apply to transparent source layers with reflecting back surface (cf., footnote 4).

Finally, we remark that in most cases, one can expect that $\langle T\rangle\left\langle\langle T\rangle_{\text {eq }}\right.$ due to the typical roll off in the transmission coefficients at large incidence angles, and the different normalization factors in (2) and (5).

\footnotetext{
${ }^{5}$ The depth of the photoexcited source of luminescence is mostly determined by the diffusion length $L$ of minority carriers. When photon recycling is negligible, the absorption length is usually longer, $\alpha L \ll 1$, and one is in the transparent limit (1). Strong photon recycling is often accompanied by enhanced effective ("photon assisted") minority-carrier diffusivity, and the opposite limit, $\alpha L \gg 1$, may prevail, so that the appropriate angular average would be (5). In the latter case, the outgoing power does not come from the entire photoexcited region, but only from its sublayer on the order of the absorption length in depth, as in (6). When neither of the two limits prevails, then the angular distribution is no longer of a universal form.

${ }^{6}$ Moreover, for transmission restricted to small angles, either averaging procedure should be also applicable to more complicated situations, characterized by "nonuniversal" angular distributions.

${ }^{7}$ Validity of (7) does not imply any prejudice as to whether (5) or (2) is the appropriate averaging procedure for a given structure. This point is made more clear when the equation is cast in the "sum rule" form (8).
}

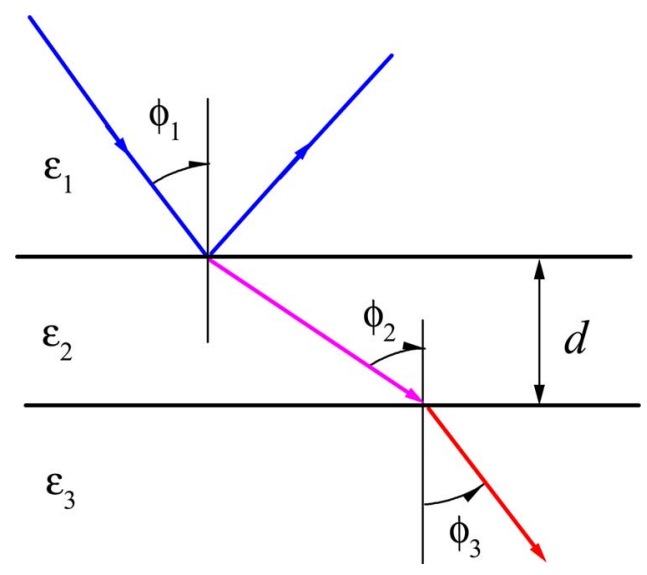

Fig. 1. Reflection and transmission of a wave incident on a homogeneous lowindex layer.

\section{TRANSPARENCY OF INTERLAYERS WITH FTIR}

In this section, we discuss the specific features due to FTIR, and focus on the angular dependences needed for calculations of the average transparencies. We consider the radiation transfer from layer 1 of permittivity $\epsilon_{1}=n_{1}^{2}$ into layer $3\left(\epsilon_{3}=n_{3}^{2}\right)$ through an intermediate layer 2, as shown in Fig. 1. We assume radiation of wavelength $\lambda$ incident on the interlayer of thickness $d$ at a fixed angle of incidence $\phi_{1}$.

Permittivity of the intermediate layer, $\epsilon_{2}=n_{2}^{2}$ is assumed real (no absorption) and lower than that of surrounding media, $\epsilon_{2}<\epsilon_{1}$, and $\epsilon_{2}<\epsilon_{3}$ with no special relation between $\epsilon_{1}$ and $\epsilon_{3}$. Exemplarily, we consider semiconductor plates, optically coupled with an optical glue [3] or a bonding oxide [6].

The reflection coefficient $R$ for small angles of incidence, $\phi_{1} \leq \phi_{t}$, is given by [10], [11]

$$
R=\frac{\left(r_{12}+r_{23}\right)^{2}-4 r_{12} r_{23} \sin ^{2} \delta}{\left(1+r_{12} r_{23}\right)^{2}-4 r_{12} r_{23} \sin ^{2} \delta}
$$

where $\delta$ is the phase shift of the wave inside the film, which is given by

$$
\delta=\frac{2 \pi d}{\lambda} \sqrt{\epsilon_{2}-\epsilon_{1} \sin ^{2} \phi_{1}}
$$

and $r_{12}$ and $r_{23}$ are the Fresnel reflection coefficients at the interfaces 1-2 and 2-3, respectively. The reflection coefficients depend on the light polarization and are given by

$$
\begin{aligned}
r_{i j, s} & =\frac{n_{i} \cos \phi_{i}-n_{j} \cos \phi_{j}}{n_{i} \cos \phi_{i}+n_{j} \cos \phi_{j}} \\
r_{i j, p} & =\frac{n_{j} \cos \phi_{i}-n_{i} \cos \phi_{j}}{n_{j} \cos \phi_{i}+n_{i} \cos \phi_{j}}
\end{aligned}
$$

where $i$ and $j$ are the interface indexes: $\{i j=12\}$ for the 1-2 interface and $\{i j=23\}$ for the 2-3 interface. Since the interlayer is assumed nonabsorbing, the transmission coefficient $T$ is readily obtained from (9) as $T=1-R$.

Equation (9) can be simplified using Fresnel's equations and Snell's refraction law, i.e., $n_{1} \sin \phi_{1}=n_{2} \sin \phi_{2}=n_{3} \sin \phi_{3}$.

After some algebra, $T$ can be written in the form

$$
T=\frac{T_{13}}{1+a T_{13} \sin ^{2} \delta}
$$



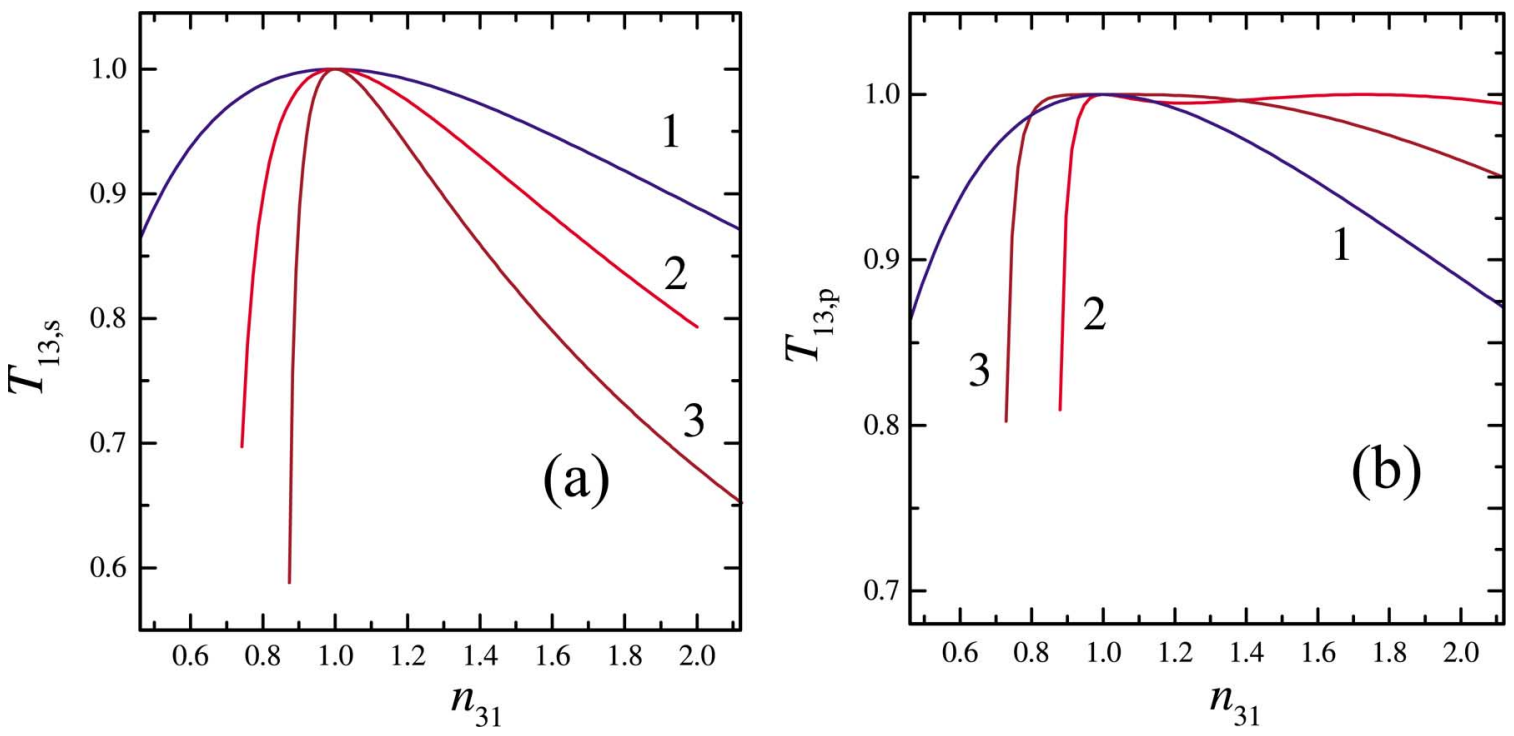

Fig. 2. (a) Interface transmission coefficients $T_{13, s}$ and (b) $T_{13, p}$ as functions of $n_{31}=n_{3} / n_{1}$ for several values of the incidence angle and two polarizations of the incident wave. 1: $\cos \phi_{1}=0 ; 2: \cos \phi_{1}=0.5 ;$ and 3: $\cos \phi_{1}=0.7$.

where $T_{13}$ is the transmission coefficient of a hypothetical interface 1-3. Away from the normal incidence, both $T_{13}$ and the coefficient $a$ are different for the two polarizations.

For $s$-polarization, we have

$$
a_{s}=\frac{\left(n_{12}^{2}-1\right)\left(n_{32}^{2}-1\right)}{4 n_{12}^{2} \cos \phi_{1}\left(1-n_{12}^{2} \sin ^{2} \phi_{1}\right) \sqrt{n_{31}^{2}-\sin ^{2} \phi_{1}}}
$$

and

$$
T_{13, s}=\frac{4 \cos \phi_{1} \sqrt{n_{31}^{2}-\sin ^{2} \phi_{1}}}{\left(\sqrt{n_{31}^{2}-\sin ^{2} \phi_{1}}+\cos \phi_{1}\right)^{2}} .
$$

Here and later, we use the notation $n_{i j}=n_{i} / n_{j}$.

For $p$-polarization, we have

$$
\begin{aligned}
a_{p}= & \frac{a_{s}}{n_{31}^{2}}\left[\left(n_{12}^{2}+1\right) \sin ^{2} \phi_{1}-1\right] \\
& \times\left[\left(n_{32}^{2}+1\right) \sin ^{2} \phi_{1}-n_{31}^{2}\right]
\end{aligned}
$$

and

$$
T_{13, p}=\frac{4 n_{31}^{2} \cos \phi_{1} \sqrt{n_{31}^{2}-\sin ^{2} \phi_{1}}}{\left(\sqrt{n_{31}^{2}-\sin ^{2} \phi_{1}}+n_{31}^{2} \cos \phi_{1}\right)^{2}} .
$$

A critical reflection angle $\phi_{t}$ is defined by $n_{1} \sin \phi_{t}=n_{2}$. For thick interlayers, $\phi_{t}$ is the critical angle of TIR. For thinner layers, $\phi_{t}$ specifies the range of incident angles $\phi_{1} \geq \phi_{t}$, where the transmission occurs due to FTIR.

Using some caution, one can apply (12) for all incident angles, including the FTIR range, $\phi_{1}>\phi_{t}$. In this range, the phase gain $\delta$ becomes imaginary, i.e., $\delta=i \delta^{\prime}$, where

$$
\delta^{\prime}=\frac{2 \pi d n_{2}}{\lambda} \sqrt{n_{12} \sin ^{2} \phi_{1}-1}
$$

while the reflection amplitudes become unimodular, $r_{i j}=\exp \left(i \delta_{i j}\right)$. The reflection phases $\delta_{i j}$ can be readily calculated using Fresnel's equations, and one obtains the transmission coefficient in the form (see [12] for a discussion)

$$
T=\frac{T_{13}}{1-a T_{13} \sinh ^{2} \delta^{\prime}}
$$

where both $T_{13}$ and $a$ are given by (13)-(16), but $a_{s}$ and $a_{p}$ become negative.

Note the peculiar nature of transmission in the FTIR region, where the reflection coefficient from the single front interface equals unity. The single-surface total reflection is affected by the interference with light reflected by the second surface. For $\phi_{1}>\phi_{t}$, the field between the two surfaces is evanescent with a penetration length that decreases with increasing $\phi_{1}$.

According to (12) and (18), for a sufficiently thin film $(\delta \ll 1$ and $\delta^{\prime} \ll 1$ ), the transmission coefficients approach $T_{13, s}$ and $T_{13, p}$. These values, in turn, depend on $n_{31}$ and $\phi_{1}$, as shown in Fig. 2. For all incident angles, the total transparency, $T_{13, s}=$ $T_{13, p}=1$, is reached only when $n_{31}=1$. Away from the exact index matching, the decrease of $T_{13, s}$ and $T_{13, p}$ is seen to be much steeper on the side $n_{31} \leq 1$. Since the exact matching of indexes of the layers 1 and 3 is rarely possible, the structures with $n_{31} \geq 1$ are preferable.

Variation of the transmission coefficients with the interlayer thickness is analyzed in Appendix II. At the critical angle, $\phi_{1}=$ $\phi_{t}$, both $T_{s}$ and $T_{p}$ are still finite and decrease at large $d$ as $1 / d^{2}$. For $s$ polarization, the transmission coefficient decreases steadily as a function of $\phi_{1}$, but reflection remains small up to $\phi_{1}=\phi_{t}$ (unless the index contrast $n_{13}$ is high).

The reflection is generally stronger for $s$ polarization than for $p$ polarization, where it is partially suppressed due to Brewster's effect: for incidence, $\phi_{1}$ at the Brewster angle, $\phi_{B}=$ $\arctan \left(n_{21}\right)$, one has $a_{p}=0$ [from (15)] and the transmission $T_{p}=T_{13, p}$ (which equals unity for $\epsilon_{3}=\epsilon_{1}$ ).

For nonmatching layers $\left(\epsilon_{3} \neq \epsilon_{1}\right)$, there is a second Brewster angle, $\phi_{B, 2}=\arctan \left(n_{32}\right)$, corresponding to the wave in layer 2, propagating at the angle $\phi_{2}$. One has $a_{p}=0$ when $\phi_{2}=\phi_{B, 2}$, and the transmission increases to $T_{13, p}$ again. In 

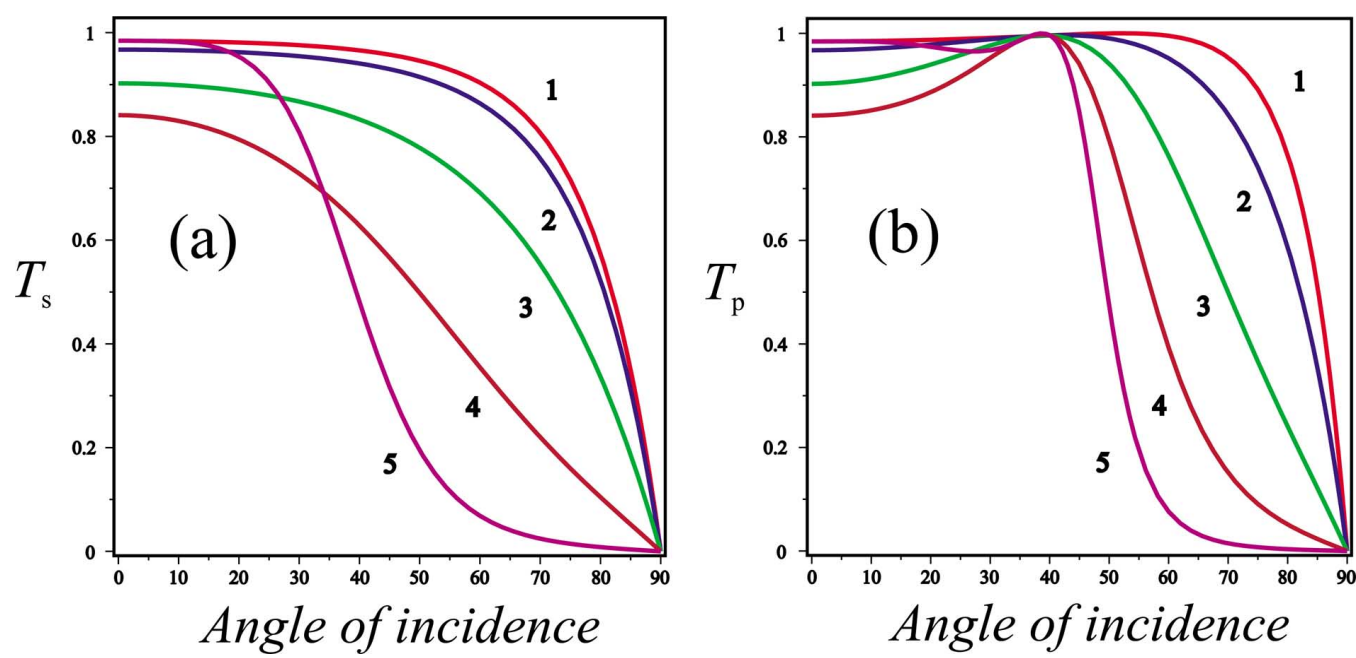

Fig. 3. Angular dependence of the transmission coefficients $T_{s}$ and $T_{p}$ for a thin layer of index $n_{2}=2.6$; the incident radiation from layer 1 with $n_{1}=3.5$ is transmitted into layer 3 with $n_{3}=4.5$. Different curves correspond to different film thicknesses described by a dimensionless parameter $\tilde{d}=d / \lambda$, viz., 1 : $\tilde{d}=0$; 2: $\tilde{d}=0.02 ; 3: \tilde{d}=0.05 ; 4: \tilde{d}=0.2$, and $5: \tilde{d}=1 /\left(2 n_{2}\right)$. The dependence on the angle of incidence is displayed in the range $0<\phi_{1}<90^{\circ}$ for two polarizations of incident light: $s$ polarization (b) and $p$ polarization. The TIR angle $\phi_{t}=48^{\circ}$, and Brewster's angles are $\phi_{B}=36.6^{\circ}$ for the $1-2$ interface and $\phi_{B}^{\prime}=40^{\circ}$ for the $2-3$ interface.

terms of the incident wave angle $\phi_{1}$, this corresponds to $\phi_{B}^{\prime}=$ $\arcsin \left(n_{31} / \sqrt{n_{32}^{2}+1}\right)$. In the range of incidence angles $\phi_{B}<$ $\phi_{1}<\phi_{B}^{\prime}$ (for $n_{31}>1$ ) or $\phi_{B}^{\prime}<\phi_{1}<\phi_{B}$ (for $n_{31}<1$ ), one has $a_{p}<0$ and $T_{p}>T_{13, p}$. When $n_{31}<1$, the transmission coefficient $T$ vanishes (and so does $T_{13}$ ) for $\sin \phi_{1} \geq n_{31}$ due to the TIR from layer 3 .

Finally, the transmission $T$ also decreases linearly with $d$ for a thin interlayer with a finite absorption coefficient $\alpha$. However, for $\alpha \lambda^{2} /\left(\pi n_{2}\right)^{2} \ll d$, this is a negligible effect [13].

\section{A. Examples: Higher Index Overlayer}

For quantitative estimates, we consider the transparency of an interlayer in a mid-IR LED optopair comprising a LED and a lens attached to the diode by a layer of optcal glue [3]. The semiconductor structure of interest consists of two semiconductor layers (InAs and CdSb) having $n_{1}=3.5$ and $n_{3}=4.5$, optically separated by a layer of a chalcogenide glass with $n_{2}=2.6$.

Calculated angular dependences of the transmission coefficient for two polarizations and several values of the layer thickness $d$ are shown in Fig. 3 in the range $0<\phi_{1}<90^{\circ}$. In this example, Brewster's angles are $\phi_{B}=36.6^{\circ}$ for surface $1-2$ and $\phi_{B}^{\prime}=40^{\circ}$ for surface $2-3$, so that the reflection of $p$-polarized light is strongly suppressed even in the region close to the TIR angle of the 1-2 interface, $\phi_{t}=48^{\circ}$.

For $\phi_{1} \rightarrow 90^{\circ}$, the reflection increases to unity. A thin interlayer approximation $T=T_{13}$ works reasonably well for very thin interlayers (compare curves $\tilde{d}=0$ and $\tilde{d}=0.02$ in Fig. 3 ), but not for thicker films [see curves $\tilde{d}=0.1$ and $\tilde{d}=1 /\left(2 n_{2}\right)$ ]. The latter case corresponds to a half-wave plate, which is seen to improve the transparency only in a range of incident angles below the TIR from the 1-2 interface.

Fig. 4 shows the average transmission coefficients $\langle T\rangle$ and $\langle T\rangle_{\text {eq }}$ calculated for this structure. Maximum values of the average transmission coefficients at $d=0$ are $\langle T\rangle_{\max }=0.836$ and $\langle T\rangle_{\text {eq, } \max }=0.94$. In the range $0<d<0.15$, the decrease of the average transmission coefficients with $d$ is almost linear.

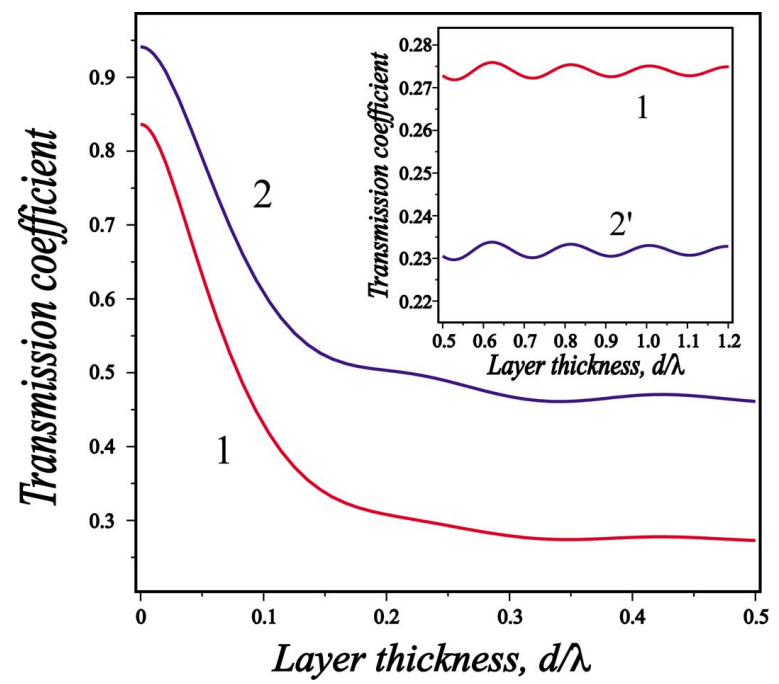

Fig. 4. Average transmission coefficients $\langle T\rangle$ (denoted as 1 ) and $\langle T\rangle_{\text {eq }}$ (denoted as 2) of a film as functions of the film thickness in units of wavelength $\lambda$ for the structure specified in Fig. 3. (Inset) Dependences $\langle T\rangle$ and $\langle T\rangle_{\text {eq }} / 2$ (denoted as $2^{\prime}$ ) at larger thickness values.

The range of quadratic decrease that could be anticipated (see Appendix II) from (12) and (18) is extremely narrow. Mathematically, this follows from the fact that the function $1 /(1+$ $a \sin ^{2} \delta$ ) decreases with $d$ almost linearly starting with $\delta \approx 0.2$ for all values of $a$ (i.e., all incidence angles), and this decrease retains its linear form after angular averaging. Thus, for both polarizations, we have a linear rather than quadratic decrease of $\langle T\rangle$ with $d$.

For $d / \lambda>\pi / n_{2}$, the dependence on $d$ becomes quite weak [see Fig. 4 (inset)] and the difference $\langle T\rangle_{\mathrm{eq}} / 2-\langle T\rangle$ becomes small. In this region, the TIR strongly restricts the angular range of interlayer transparency. Therefore, the average transmission does not vary with thickness except for a weak interference modulation. The value of the average transmission coefficient 

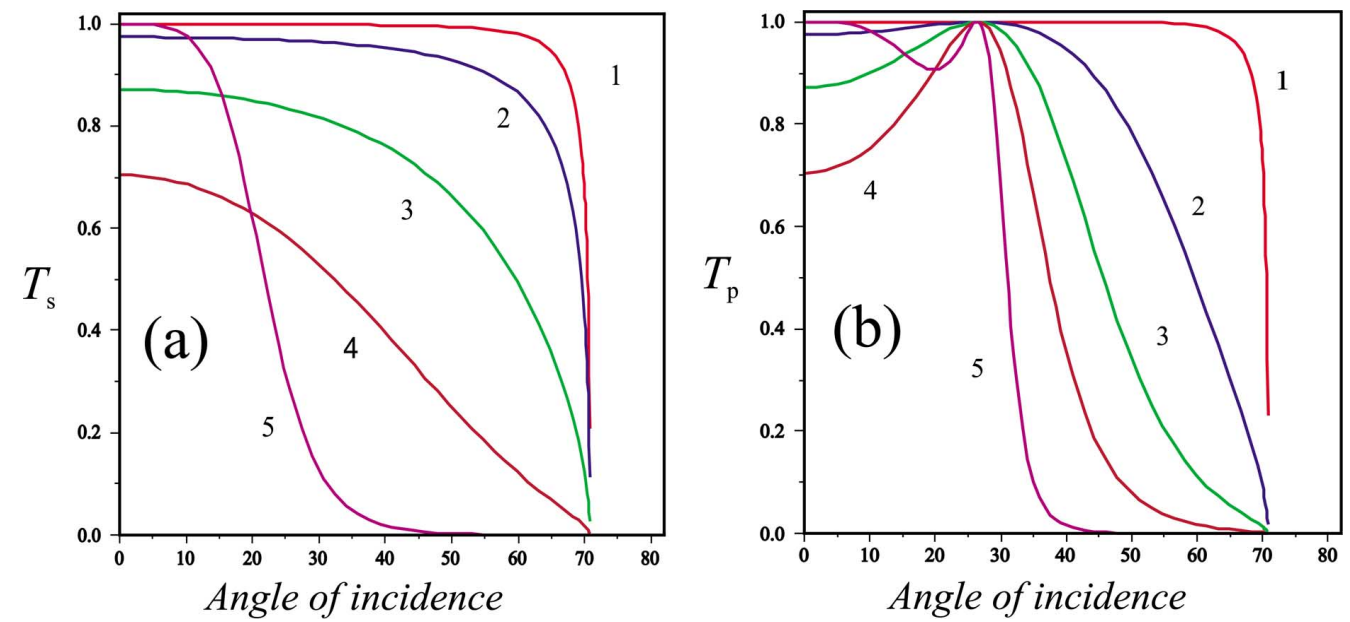

Fig. 5. Angular dependence of the transmission coefficients: (a) $T_{s}$ and (b) $T_{p}$ of a thin interlayer with refractive index $n_{2}=1.8$ (oxide) for the radiation incident from the first layer with $n_{1}=3.6(\mathrm{GaAs})$ and transmitted into the third layer with $n_{3}=3.4(\mathrm{InP})$. Different curves correspond to different (dimensionless) interlayer thicknesses $\tilde{d}=d / \lambda$ viz., $1: \tilde{d}=0 ; 2: \tilde{d}=0.02 ; 3: \tilde{d}=0.05 ; 4: \tilde{d}=0.1$; and $5: \tilde{d}=1 /\left(2 n_{2}\right)$. The TIR angle $\phi_{t}=30^{\circ}$, and the Brewster angles are $\phi_{B}=26.6^{\circ}$ and $\phi_{B}^{\prime}=26.2^{\circ}$.

can be estimated by taking $\sin ^{2} \delta \approx 1 / 2$ in (12) and restricting the range of angular integration to $0<\phi_{1}<\phi_{t}$. This gives $\langle T\rangle=0.27$ and $\langle T\rangle_{\text {eq }}=0.45$.

Due to the TIR phenomenon, it is very difficult to obtain high power transmission across a low-index interlayer. The frustration of TIR helps, but not very much, unless the interlayer is exceedingly thin. For example, to obtain an average interlayer transmission $\langle T\rangle \geq 60 \%$, one needs to take $d / \lambda \leq 0.05$. For the mid-IR region $(\lambda=3 \mu \mathrm{m})$, this gives $d \leq 0.15 \mu \mathrm{m}$, which is quite challenging technologically.

Another important area of the interlayer application is optical integration of different semiconductors via an interfacial oxide layer. In light of our results, even the best (thinnest) reported oxide layers, used for optical wafer bonding, may not be sufficiently thin for some of the intended applications. Consider the case of an InP photodiode $\left(n_{1}=3.4\right)$ structure bonded with $\mathrm{SiO}_{2}$ bonding layer of index $n_{2}=1.46$ to a $\mathrm{Si}$ wafer $\left(n_{3}=3.45\right)$. Even for the recently reported [6] record-thin $60 \mathrm{~nm}$ oxide bonding layer, one has $\tilde{d} \approx 0.1$, resulting in a strong reflection for a wide range of angles. To ensure reasonable power transmission across the bonding layer, one would have to lower its thickness $d$ well under $\lambda /\left(2 \pi n_{2}\right) \approx \lambda / 12$, which is again quite challenging.

Besides pursuing still thinner interlayers, one can circumvent the limitations presented by TIR by making the source layer a resonant cavity with highly anisotropic emission. The cavity case goes beyond the scope or our analysis.

\section{B. Examples: Lower Index Overlayer}

Another practical example is a bonded photodetector on top of a semiconductor scintillator slab with an optical glue in between. We consider a semiconductor structure consisting of a scintillator layer 1 (GaAs, emission $\lambda=860 \mathrm{~nm}$, index $n_{1}=$ $3.6)$ and a photodiode layer $3\left(\operatorname{InP}, n_{3}=3.4\right)$. For the near-IR region, the refractive index of the available optical glues does not exceed $n \approx 2$. Here, we assume that the layers are optically separated by an interlayer with a typical index $n_{2}=1.8$. Fig. 5

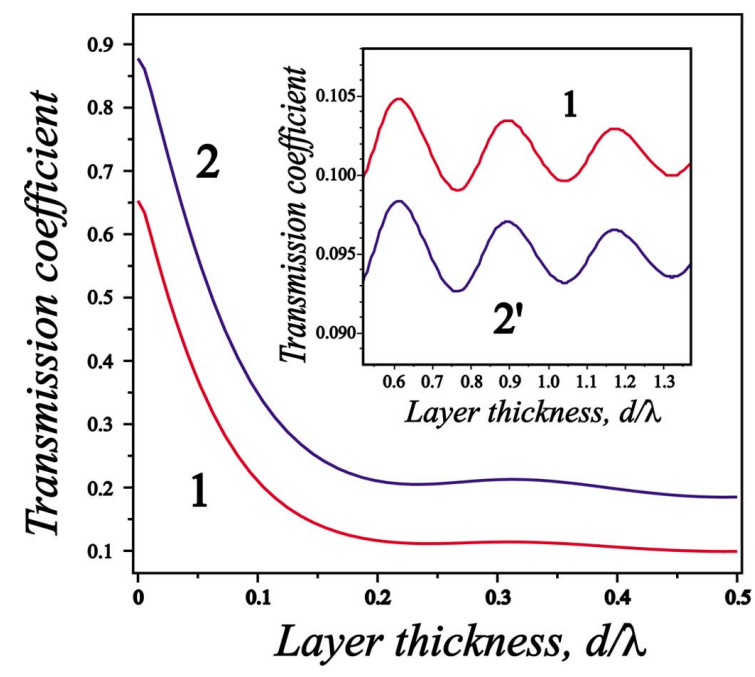

Fig. 6. Average transmission coefficients $\langle T\rangle$ (denoted as 1 ) and $\langle T\rangle_{\text {eq }}$ (denoted as 2) of a film as functions of the film thickness in units of wavelength $\lambda$ for the structures specified in Fig. 5. (Inset) Dependences $\langle T\rangle$ and $\langle T\rangle_{\text {eq }} / 2$ (denoted as $2^{\prime}$ ) for thicker layers.

shows the transmission coefficients $T_{13, s}$ and $T_{13, p}$, calculated as functions of the incidence angle for different interlayer thicknesses. Due to the high-index contrast, the TIR angle from the interlayer is only $30^{\circ}$. The smaller index of layer $3\left(n_{31}=0.94\right)$ results in an additional TIR and reduces the overall transparency region to $\phi_{1} \leq 70.8^{\circ}$. Due to the tight matching of $n_{1}$ and $n_{3}$ indices, the reflection losses could be small for sufficiently thin interlayers. However, requirements to their thickness are quite stringent, especially in the near-IR region. Even at small angles of incidence, one should use films with $d / \lambda<0.05$ in order to reduce reflection losses below $10 \%$. For the chosen $\lambda=860 \mathrm{~nm}$, this corresponds to $d \leq 50 \mathrm{~nm}$, which is extremely challenging.

Fig. 6 shows the average transmission coefficients $\langle T\rangle$ and $\langle T\rangle_{\text {eq }}$ calculated for the structures specified in Fig. 5. The maximum achievable average transmission coefficient is only $\langle T\rangle=$ 
$\left\langle T_{13}\right\rangle=65 \%$, and the drop compared with the LED case considered earlier resulting from the lesser overlayer index. Again, the decrease of the average transmission coefficients with $d$ at $d / \lambda \ll 0.15$ is not proportional to $d^{2}$, but almost linear. Due to the smaller angle $\phi_{t}$, the averaging over $\phi$ smears the interference picture in the transmission to a lesser extent. The values of transmission coefficient at $d / \lambda \geq 0.15$, estimated using the same approximation as in the case of high-index interlayer, are $\langle T\rangle=0.096$ and $\langle T\rangle_{\text {eq }}=0.18$.

The low value of $\langle T\rangle$, which results from the high $n_{12}$ contrast, makes the range $d / \lambda \geq 0.15$ practically unsuitable for the scintillator-photodiode pair application. Pursuing much thinner intermediate layers may be very difficult. In our view, the more promising approach to combine a GaAs scintillator with an InP photodiode in one integrated detector would be to use the wafer fusion technique [14], [15]—which dispenses with an intermediate bonding layer altogether.

\section{DISCUSSION AND CONCLUSION}

We have analyzed the transparency of low-index intermediate layers between two higher index semiconductor layers in the situation when the angular spread of incident radiation is important. We paid special attention to the angular average of the optical power transmission, and showed that for two practically relevant classes of source layers, it can be cast in a universal form. These classes include both highly transparent layers and absorbing layers with homogeneously distributed emission sources over the thickness exceeding the absorption length. Our results are also relevant to the interpretation of common luminescence experiments.

Transmission of isotropic radiation through low-index interlayers $\left(n_{2}\right.$ in the range 1.5-2.5) is found to decrease almost linearly in the interval $0 \leq d \lesssim 0.1 \lambda$ from its value at $d=0$ to a practically constant value at $d \gtrsim 0.2 \lambda$. This result is valid for all practical three-layer semiconductor structures, as has been checked numerically for the exemplary structures with the indexes of refraction $n_{1}$ and $n_{3}$ in the range $n=3-4$.

For the typical thicknesses $(d \gtrsim 1 \mu \mathrm{m}$ ) of the optical glue interlayers, $d \gtrsim 0.2 \lambda$, the reflection losses are high even in the mid-IR region, and grow from $\approx 70 \%$ to $\geq 90 \%$ with increasing index contrast between the source layer and the interlayer. This happens because the average transparency is now mostly governed by the narrowing escape angle $\phi_{t}$ from TIR. Therefore, the transmission does not depend on the layer thickness $d$, and can be estimated using the simplified approach that neglects the interference effects and the FTIR.

For thinner interlayers $d \leq 0.1 \lambda$ with low refractive index, the transmission depends on the frustrated total internal reflection due to the constructive interference of evanescent waves reflected by the two surfaces of the interlayer. The main contribution to the average optical power transmission comes from fairly large angles of incidence, implying a less significant reflection loss. Quantitative analysis of the optical power transmission across low-index intermediate layers therefore requires both careful angular averaging and accurate evaluation of the transmission coefficients in the FTIR range.
Finally, we have found a simple and universal sum rule that must be satisfied by the angular dependence of an optical power transmission coefficient between two media. The sum rule has the form of a reciprocal relation (8) and holds for any three-layer structures with a nonabsorbing interlayer of arbitrary thickness and refractive index. The only limitation on the validity of sum rule (8) arises from the requirement that reflections from the back surfaces of both the source and the receiving layers be absent, for example, when the thicknesses of these layers exceed their absorption lengths. The sum rule explicitly demonstrates that the average transmission coefficient of the three-layer structure is sensitive to the overlayer refractive index relative to that of the source layer, and that structures with larger overlayer index are preferable.

\section{APPENDIX I}

\section{EQUILIBRIUM RADIATION AND TRANSMITTED LUMINESCENCE}

Here, we consider the effects of the surface transparency on the thermal emission by a homogeneous and optically isotropic material. The rate of radiation emission obeys the detailed balance between the emission and absorption processes embodied in the van Roosbroeck-Shockley relation [16], and is proportional to the absorption coefficient $\alpha(\omega)$. At a temperature $T$, the photon density in the unit frequency interval at $\omega$ emitted in unit volume per unit time is given by [1]

$$
N_{\omega}=\frac{n_{1}^{2} \omega^{2} \alpha(\omega)}{\pi^{2} c^{2}[\exp (\hbar \omega / k T)-1]} .
$$

On its way out, the radiation may be absorbed and re-emitted many times, but this does not change the equilibrium photon density. The number of photons reaching the surface unit at distance $r$ from the emitting source in volume $d v$ equals

$$
d I(r)=\frac{1}{4 \pi r^{2}} \exp (-\alpha r) N_{\omega} d v
$$

Therefore, the total photon flux $\left.I\right|_{z=0}$ to the unit surface (at $z=0$ ) from the region $z>0$ in a unit solid angle about a fixed incidence angle $\phi_{1}$ can be obtained by integration over $r$, which takes the form

$$
\left.I_{\omega}\right|_{z=0}=\frac{c_{1} P_{\omega}}{4 \pi} \int_{0}^{\infty} \exp \left(-\frac{\alpha z}{\cos \phi_{1}}\right) \frac{\alpha}{\cos \left(\phi_{1}\right)} d z
$$

where $P_{\omega}=N_{\omega} /\left(\alpha c_{1}\right)$ is the equilibrium photon density of thermal radiation and $c_{1}=c / n_{1}$ is the speed of light in the emitting material. Since the integral on the right-hand side of (A1.3) equals unity, (A1.3) shows that the equilibrium flux to the surface is identical to blackbody radiation, and depends neither on the incidence angle nor on the particular shape of $\alpha(\omega)$ (weak emission rate in frequency regions of small absorption is compensated by the high material transparency at these frequencies).

The energy transmitted to material 3 is given by the integral over the normal component of the incident flux, multiplied by the transmission coefficient $T_{13}$, i.e.,

$$
Q_{31}=\int T_{13} \cos \phi_{1} I_{z=0} d \Omega
$$


where we have suppressed the frequency index $\omega$ since (A1.4) is valid for each frequency individually. For $T_{13}=1$, it gives a well-known result $Q_{31}=c_{1} P_{\omega} / 4$. For $T_{13} \neq 1$, we have

$$
Q_{31}=\left\langle T_{13}\right\rangle_{\mathrm{eq}} c_{1} \frac{P_{\omega}}{4}
$$

where the averaging of the transmission coefficient from 1 to 3 is defined by (5). It is readily seen that (6) and (A1.5) are equivalent.

Consider the case of a thermal equilibrium between material layers 1 and 3 with any intermediate layer 2 . For the equilibrium to hold, one should have $Q_{13} \equiv Q_{31}$. This requires that

$$
n_{1}^{2}\left\langle T_{13}\right\rangle_{\mathrm{eq}}=n_{3}^{2}\left\langle T_{31}\right\rangle_{\mathrm{eq}}
$$

and therefore, (8) must hold. Suppose $n_{1}>n_{3}$. Part of the equilibrium radiation incident on the interface gets reflected due to the TIR phenomenon. However, this is exactly compensated by the higher density of photon states in the higher index material. This compensation is "moderated" by the slower velocity of the energy flux in the second material, so that the resultant compensating effect is of the second, and not the third, power in $n_{13}$.

The sum rule expressed by (A1.6) and (8) remains valid in a nonequilibrium situation when there is no radiation compensation. It also holds in the case of arbitrary small absorption coefficients in layers 1 and 3-so long as there are no back mirrors that would make the problem of transmission of a single incident wave irrelevant. It holds for any planar interface, including any intermediate layer of index $n_{2}$, so long as there is no absorption in the intermediate layer. This can be verified by direct inspection of the integrals using explicit expressions (12) for both polarizations. To do this, we note that the transmission coefficients $T_{13}$ and $T_{31}$ can be written as functions of both the incidence angle and the refraction angle, subject to Snell's law $n_{1} \sin \phi_{1}=n_{3} \sin \phi_{3}$, which holds for both directions of transmission. As an example, we can write $T_{13, s}$ in the form

$$
T_{13, s}=\frac{4 n_{1} n_{3} \cos \phi_{1} \cos \phi_{3}}{\left(n_{3} \cos \phi_{3}+n_{1} \cos \phi_{1}\right)^{2}}
$$

which makes symmetry between transmission coefficients $1 \rightarrow$ 3 and $3 \rightarrow 1$ evident. Similarly, $a_{s}$ can be written as $a_{s}=$ $b_{12} b_{32}$, where

$$
b_{i j}=\frac{\left(n_{i}^{2}-n_{j}^{2}\right)}{2 n_{i} \cos \phi_{i} \sqrt{n_{j}^{2}-n_{i}^{2} \sin ^{2} \phi_{i}}}
$$

and the phase shift as

$$
\delta=\frac{2 \pi d / \lambda}{\left(n_{2}^{2}-n_{1}^{2} \sin ^{2} \phi_{1}\right)^{1 / 4}\left(n_{2}^{2}-n_{3}^{2} \sin ^{2} \phi_{3}\right)^{1 / 4}} .
$$

Then, one can replace integration on the left-hand side of (8) over $\phi=\phi_{1}$ by integration over $\phi_{3}$, so that $n_{1} \cos \phi_{1} d \phi_{1}=$ $n_{3} \cos \phi_{3} d \phi_{3}$ or, with Snell's law,

$$
n_{1}^{2} \cos \phi_{1} \sin \phi_{1} d \phi_{1}=n_{3}^{2} \cos \phi_{3} \sin \phi_{3} d \phi_{3} .
$$

Finally, changing the integration interval, we arrive at a direct proof of the sum rule (8) for each polarization.

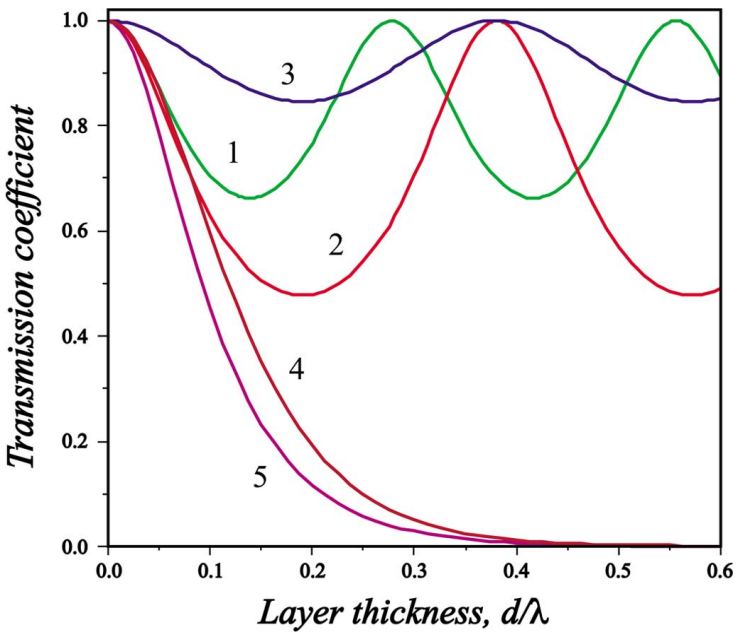

Fig. 7. Dependence of the transmission coefficients $T_{s}$ and $T_{p}$ of a film on the dimensionless layer thickness $\tilde{d}=d / \lambda$. The structure parameters are the same as in Fig. $5\left(\phi_{t}=30^{\circ}\right)$. Different curves correspond to different values of the angle of incidence and different polarizations of the incident light viz., 1: $\phi_{1}=0 ; 2: \phi_{1}=20^{\circ} s ; 3: \phi_{1}=20^{\circ} p ; 4: \phi_{1}=36^{\circ} s ;$ and $5: \phi_{1}=36^{\circ} p$.

\section{APPENDIX II \\ TRANSMISSION COEFFICIENT AT SELECTED ANGLES OF INCIDENCE}

The calculated dependence of the transmission coefficients on the interlayer thickness in the structure with lower index overlayer $\left(n_{31}<1\right)$ is shown in Fig. 7 for two polarizations and several angles of incidence, including the normal incidence and representative angles below and above $\phi_{t}$.

For $\phi_{1}<\phi_{t}$, we see maxima in the transmission coefficient for both polarizations. These maxima are due to constructive interference between the waves in the middle layer and occur at $\delta=\pi m$, where $m$ is an integer $(m=1,2, \ldots)$. The interference structure depends on the angle of incidence (for normal incidence, the maxima correspond to $d=m \lambda / 2 n_{2}$ ). The antireflection effect arises for half-wave plates-rather than quarter-wave plates, which would be the case in structures with either $n_{1}<n_{2}<n_{3}$ or $n_{1}>n_{2}>n_{3}$. The almost total transparency of the film at the interference maxima is due to the phase shift in the reflection from the two film surfaces, which results in $r_{12} \approx-r_{23}$, and makes the interlayer "an absentee layer" (the transmission coefficients become $T_{13, s}$ and $T_{13, p}$, given by (14) and (16), respectively).

In the FTIR range, $\phi_{1}>\phi_{t}$, the transmission coefficients roll off rapidly with the thickness $d$, and this roll-off becoming exponential for $d \geq \lambda / n_{2}$.

To further clarify the dependences displayed in Fig. 7, we discuss next some detail of our calculations for $\phi_{1} \leq \phi_{t}$. These results follow from the general formulas of Section III. For normal incidence, one has $T_{13, s}=T_{13, p}=T_{13}$, where

$$
\begin{aligned}
T_{13} & =\frac{4 n_{31}}{\left(1+n_{31}\right)^{2}} \\
R_{13} & =\frac{\left(1-n_{31}\right)^{2}}{\left(1+n_{31}\right)^{2}}
\end{aligned}
$$


The ratio $n_{31}$ is close to unity for typical semiconductor pairs, e.g., taking both indexes in the range of 3.5-4, we find that the reflection losses at normal incidence do not exceed $0.5 \%$.

For an optically thin film, $\delta_{0} \ll 1$, and from (12), one has

$$
\left.T\right|_{\phi_{1}=0}=T_{13}-4 T_{13}^{2} \frac{\sqrt{R_{12} R_{23}}}{T_{12} T_{23}}\left(2 \pi n_{2} \tilde{d}\right)^{2}
$$

where $\tilde{d}=d / \lambda$, and the reflection and transmission coefficients for the 1-2 and 2-3 interfaces are defined similar to $R_{13}, T_{13}$ in (A2.1). We see that the deviation $T-T_{13} \propto \tilde{d}^{2}$. For $\phi_{1} \geq \phi_{t}$, we reach the same conclusion by expanding in powers of $\tilde{d}$ the expression (18).

Physically, the decrease of $T$ is quadratic because it is proportional both to the layer thickness and the fraction of the wavelength that is localized in it. Similar effect is well known in quantum mechanics [17], [18], where the particle transmission coefficient through a thin quantum well is of the form quite similar to (12) with $n_{31}=1$

$$
T=\frac{1}{1+a \sin ^{2} \delta}
$$

where $\delta=k d$ is the wavefunction phase shift in the well and $a$ is a constant proportional to the square of the well depth. For transmission through a barrier, $\delta=i \delta^{\prime}$ and $a$ changes its sign so that (A2.3) becomes similar to (18). In both cases, $T$ deviates from unity by an amount proportional to the square of a product of potential perturbation and its thickness.

For $\phi_{1} \rightarrow \phi_{t}$, the Fresnel reflection coefficients tend to unity with different signs viz., $r_{12, s}=r_{12, p} \rightarrow 1$ and $r_{23, s}=r_{23, p} \rightarrow$ -1 . At the same time, one has $\delta \rightarrow 0$. Therefore, to calculate the transparency in the vicinity of $\phi_{1}=\phi_{t}$, one needs a more accurate evaluation of the limit $\phi_{1} \rightarrow \phi_{t}$ in (9) and (12). It is convenient to give expressions for the reciprocal transparencies in terms of layer permittivities viz.

$$
\begin{aligned}
\left.\frac{1}{T_{s}}\right|_{\phi_{1}=\phi_{t}=} & \frac{\left(\sqrt{\epsilon_{1}-\epsilon_{2}}+\sqrt{\epsilon_{3}-\epsilon_{2}}\right)^{2}}{4 \sqrt{\epsilon_{1}-\epsilon_{2}} \sqrt{\epsilon_{3}-\epsilon_{2}}} \\
& +\pi^{2} \tilde{d}^{2} \sqrt{\epsilon_{1}-\epsilon_{2}} \sqrt{\epsilon_{3}-\epsilon_{2}} \\
\left.\frac{1}{T_{p}}\right|_{\phi_{1}=\phi_{t}=} & \frac{\left(\epsilon_{3} \sqrt{\epsilon_{1}-\epsilon_{2}}+\epsilon_{1} \sqrt{\epsilon_{3}-\epsilon_{2}}\right)^{2}}{4 \epsilon_{1} \epsilon_{3} \sqrt{\epsilon_{1}-\epsilon_{2}} \sqrt{\epsilon_{3}-\epsilon_{2}}} \\
& +\pi^{2} \tilde{d}^{2} \sqrt{\epsilon_{1}-\epsilon_{2}} \sqrt{\epsilon_{3}-\epsilon_{2}} .
\end{aligned}
$$

For matching layers, $\epsilon_{3}=\epsilon_{1}$, and $s$-polarized waves, we have

$$
\left.T_{s}\right|_{\phi_{1}=\phi_{t}}=\frac{1}{1+\pi^{2} \tilde{d}^{2}\left(\epsilon_{1}-\epsilon_{2}\right)} .
$$

Similarly, for matching layers and $p$-waves, we have

$$
\left.T_{p}\right|_{\phi_{1}=\phi_{t}}=\frac{1}{1+\pi^{2} \tilde{d}^{2}\left(\epsilon_{1}-\epsilon_{2}\right)\left(\epsilon_{2} / \epsilon_{1}\right)^{2}} .
$$

Thus, at $\phi_{1}=\phi_{t}$, the transmission coefficient remains finite and steadily, but not exponentially, decreases with increasing thickness of the intermediate layer.

\section{ACKNOWLEDGMENT}

The authors would like to thank Dr. Suchalkin for his useful discussions.

\section{REFERENCES}

[1] T. S. Moss, G. J. Burrell, and B. Ellis, Semiconductor Opto-Electronics. London, U.K.: Butterworth, 1973.

[2] D. Delbeke, R. Bockstaele, P. Bienstman, R. Baets, and H. Benisty, "High-efficiency semiconductor resonant-cavity light-emitting diodes: A review," IEEE J. Sel. Topics Quantum Electron., vol. 8, no. 2, pp. 189-206, Mar./Apr. 2002.

[3] M. A. Remennyi, N. V. Zotova, A. A. Kardashev, B. A. Matveev, N. M. Stus', and G. N. Talalakin, "Low voltage episide down bonded mid-IR diode optopairs for gas sensing in the 3.3-4.3 $\mu \mathrm{m}$ spectral range," Sens. Actuators B, Chem., vol. 91, no. 1-3, pp. 256-261, Jun. 2003.

[4] H. Gauck, T. H. Gfroerer, M. J. Rean, E. A. Cornell, and K. A. Bertness, "External radiative quantum efficiency of $96 \%$ from a GaAs/GaInP heterostructure," Appl. Phys. A, Mater. Sci. Process., vol. 64, no. 2, pp. 143-147, Jan. 1997.

[5] A. A. Kastalsky, S. Luryi, and B. Spivak, "Semiconductor high-energy radiation scintillation detector," Nucl. Instrum. Methods Phys. Res. A, Accel. Spectrom. Detect. Assoc. Equip., vol. 565, no. 2, pp. 650-656, Sep. 2006.

[6] D. Liang, A. W. Fang, H. Park, T. E. Reynolds, K. Warner, D. C. Oakley, and J. E. Bowers, "Low temperature, strong $\mathrm{SiO}_{2}-\mathrm{SiO}_{2}$ covalent wafer bonding for III--V compound semiconductors-to-silicon photonic integrated circuits," J. Electron. Mater., vol. 37, no. 10, pp. 1552-1559, Oct. 2008.

[7] S. Zhu, A. W. Yu, D. Hawley, and R. Roy, "Frustrated total internal reflection: A demonstration and review," Amer. J. Phys., vol. 54, no. 7, pp. 601-607, Jul. 1986.

[8] L. D. Landau and E. M. Lifshitz, Statistical Physics, 3rd ed. Oxford, U.K.: Pergamon, 1984, pt. I.

[9] I. Schnitzer, E. Yablonovitch, C. Caneau, and T. J. Gmitter, "Ultrahigh spontaneous quantum efficiency, $99.7 \%$ internally and $72 \%$ externally, from $\mathrm{AlGaAs} / \mathrm{GaAs} / \mathrm{AlGaAs}$ double heterostructures," Appl. Phys. Lett., vol. 62, no. 2, pp. 131-133, Jan. 1993.

[10] M. Born and E. Wolf, Principles of Optics. New York: Pergamon, 1980, sec. Sec 1.6.4.

[11] L. D. Landau and E. M. Lifshitz, Electrodynamics of Continuous Media. Oxford, U.K.: Pergamon, 1984.

[12] I. N. Court and F. K. Willisen, "Frustrated total internal reflection and application of its principle to laser cavity design," Appl. Opt., vol. 3, no. 6, pp. 719-726, Jun. 1964

[13] F. Abelès, "Optical properties of thin absorbing films," J. Opt. Soc. Amer., vol. 47, no. 6, pp. 473-482, Jun. 1957.

[14] A. Mereuta, V. Iakovlev, A. Caliman, A. Syrbu, P. Royo, A. Rudra, and E. Kapon, "In(Al)GaAs-AlGaAs wafer fused VCSELs emitting at 2- $\mu \mathrm{m}$ wavelength," IEEE Photon. Technol. Lett., vol. 20, no. 1, pp. 24-26, Jan. 2008.

[15] L. Sagalowicz, A. Rudra, E. Kapon, M. Hammar, F. Salomonsson, A. Black, P.-H. Jouneau, and T. Wipijewski, "Defects, structure, and chemistry of InP-GaAs interfaces obtained by wafer bonding," J. Appl. Phys., vol. 87, no. 9, pp. 4135-4146, May 2000.

[16] W. van Roosbroeck and W. Shockley, "Photon-radiative recombination of electrons and holes in germanium," Phys. Rev., vol. 94, no. 6, pp. 1558-1560, Jun. 1954.

[17] P. M. Mathews and K. Venkatesan, A Textbook of Quantum Mechanics. New York: McGraw-Hill, 1978, p. 62.

[18] M. Visser, "Some general bounds for one-dimensional scattering," Phys. Rev. A, Gen. Phys., vol. 59, no. 1, pp. 427-438, Jan. 1999.

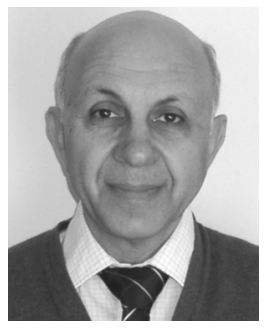

Arsen V. Subashiev received the M.Sc. degree in optoelectronics from St. Petersburg State Polytechnic University, St. Petersburg, Russia, and the Ph.D. degree in physics and mathematics from Ioffe PhysicoTechnical Institute, St. Petersburg, in 1969.

He was with Ioffe until 1978. He was a Professor in the Department of Experimental Physics, St. Petersburg State Polytechnic University. He is currently a Research Professor in the Department of Electrical and Computer Engineering, State University of New York, Stony Brook. He is the author or coauthor of more of more 90 papers published in the field of theory of electric, photoelectric, and optical phenomena in semiconductors and semiconductor devices. His research interests include the theory of semiconductors and semiconductor nanostructures, theory of spin-polarized electron emission, optoelectronics, and photonics. 


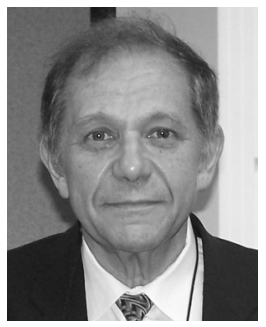

Serge Luryi (M'81-SM'85-F'90) received the Ph.D. degree in theoretical physics from the University of Toronto, Toronto, ON, Canada, in 1978.

In 1980, he joined Bell Laboratories, where he was practicing research in physics of semiconductor devices, and was a Group Supervisor and a Distinguished Member of the Technical Staff until 1994. In 1994, he joined the State University of New York, Stony Brook, where he is currently the Chair of the Department of Electrical and Computer Engineering. $\mathrm{He}$ is also the Founding Director of the NY State Center for Advanced Sensor Technology. He is the author or coauthor of more than 200 scientific papers published in various international journals. He holds 46 U.S. patents.
Dr. Luryi was the Editor-in-Chief of the IEEE TRANSACTIONS ON ELECTRON DEVICES between 1986 and 1990. In 1995, he organized the first international advanced research workshop on the "Future Trends in Microelectronics," which has since grown into a celebrated series of conferences. He was elected a Fellow of the American Physical Society during 1993 for contributions to the theory of electron transport in low-dimensional systems and invention of novel electron devices and a Fellow of the Optical Society of America during 2007 for outstanding and pioneering contributions to semiconductor optoelectronics, especially to the physics and photonic applications of low-dimensional semiconductor structures. In 2003, he was appointed to the rank of Distinguished Professor by the Board of Trustees of SUNY. He was the recipient of the 2006 IEEE Papoulis Award for Excellence in Engineering and Technology Education for pioneering contributions to include entrepreneurial skills in engineering education on Long Island. 